Fecha de recepción: 25 de agosto del 2017

Fecha de aceptación: 7 de noviembre del 2017

\title{
ABP CON EL USO DEL BLENDED LEARNING PARA MEJORAR EL APRENDIZAJE DE TENDENCIA CENTRAL Y DISPERSIÓN
}

\author{
ABP WITH THE USE OF BLENDED LEARNING TO IMPROVE THE \\ LEARNING OF CENTRAL TENDENCY AND DISPERSION
}

Irene Maricela Silva Siesquén ${ }^{1}$

\section{RESUMEN}

El presente trabajo de investigación tuvo como objetivo determinar si la aplicación de la metodología del aprendizaje basado en problemas (ABP) en la modalidad blended learning mejora el aprendizaje de las medidas de tendencia central y dispersión en los estudiantes de la carrera profesional de Enfermería de la Universidad Católica los Ángeles de Chimbote, en el semestre académico 2014-02. El estudio fue de tipo cuantitativo con un diseño de investigación pre experimental con pretest y postest a un solo grupo. Se trabajó con una población muestral de 26 estudiantes del II ciclo - sección "A”. Para comprobar las hipótesis de investigación se utilizó la prueba estadística no paramétrica de Wilcoxon, con un nivel de significancia del 5\%. Se obtuvo como resultados en el pretest que el 96,2\% de los estudiantes tenían un nivel de logro de aprendizaje "malo"; en el postest un 57,7\% en el nivel "bueno" y un 30,8\% en un nivel "excelente", estos resultados permiten concluir que la aplicación de la metodología del ABP en la modalidad blended learning mejora el aprendizaje de las medidas de tendencia central y dispersión.

PALABRAS CLAVE: Blended learning, Aprendizaje basado en problemas.

1 Licenciada en Estadística. Magister en Educación con mención en Educación a Distancia. Docente de la Escuela Profesional de Enfermería - ULADECH Católica. 


\section{ABSTRACT}

This research aimed to determine whether the application of the methodology of problem based learning $(\mathrm{PBL})$ in blended learning mode improves learning of the measures of central tendency and dispersion students career of Nursing Angels Catholic University of Chimbote in 2014 - II. The quantitative study is a pre experimental research design with pretest and posttest one group. We worked with a sample population of 26 students of the second cycle "A" section. To test the research hypotheses the nonparametric Wilcoxon statistical test was used, with a significance level of 5\%. It was obtained as in the pretest results that $96.2 \%$ of students have an achievement level of "bad" learning; in the posttest $57.7 \%$ in the "good" level and $30.8 \%$ in an "excellent" level, these results suggest that the application of the methodology of PBL in blended learning mode improves learning was obtained measures of central tendency and dispersion.

KEY WORDS: Blended learning, problem-based learning.

\section{INTRODUCCIÓN}

Las metodologías de enseñanza aprendizaje en Blended Learning (blearning), conforma un conjunto de acciones presenciales, donde se presentan como una alternativa para reforzar el aprendizaje y para conseguir una mayor eficiencia en la comprensión y resolución de problemas (Hinojo, Aznar y Cáceres, 2009).

El Aprendizaje Basado en Problemas (ABP), es entendido como caso o situaciones problemas, que conducen a un estudio de casos. Es una metodología centrada en el aprendizaje, la investigación y reflexión que siguen los alumnos para llegar a una solución ante un problema planteado por el profesor.

La utilización de metodologías activas como el ABP en el proceso de enseñar y aprender, pretende promover el aprendizaje colaborativo en pequeños grupos, orientado a la solución de problemas que son diseñados en general a partir de noticias, donde se aprende investigando y buscando la información de los contenidos y de la propia experiencia de trabajar en el aula. El aprendizaje se adquiere a medida que se avanza en la resolución del problema, que es asumido por el alumno y no por el profesor, el cual juega un rol de mediador, negociador de significado. Esta forma de ABP, implica abordar un problema integrador, que es el eje conductor de la unidad en estudio; se estructura en torno a una secuencia lógica de problemas acotados a esta situación, para alcanzar la solución del problema integrador al final de la unidad (Espinoza y Sánchez, 2014). 
El ABP incluye actividades de aprendizaje de: exploración de ideas previas, introducción de variables, síntesis y transferencia de contenido, y giran en torno a la discusión de un problema (Sánchez, Moreira y Caballero, 2009; Duch, Groh y Allen, 2004).

Esta investigación se fundamenta en la línea de investigación de Escuela profesional de Educación Intervenciones didácticas. Considerando por ello nuestra propuesta "Aplicación de estrategias didácticas en ABP con el uso del blended learning para mejorar el aprendizaje de las medidas de tendencia central y dispersión en los estudiantes de la carrera profesional de enfermería de la Universidad Católica los Ángeles de Chimbote, año 2014".

Tiene como propósito indagar si la aplicación de estrategias didácticas en Blended Learning con la metodología del ABP mejora el aprendizaje de las medidas de tendencia central y de dispersión en los estudiantes de la Carrera Profesional de Enfermería de la Universidad Católica los Ángeles de Chimbote.

La investigación incluye aplicar estrategias didácticas en la plataforma moodle: Entorno Virtual Angelino (EVA) donde se tiene implementada la asignatura de estadística de acuerdo a lo planificado en el silabo y plan de aprendizaje (SPA) para la ejecución del desarrollo temático tanto de la parte teórica como práctica.

Como aporte central la presente investigación busca demostrar que el desarrollo de la asignatura de estadística en la modalidad blendend learning con apoyo de la plataforma moodle utilizando la metodología del ABP y propiciando el trabajo colaborativo contribuirá a mejorar el aprendizaje de las medidas de tendencia central y de dispersión que integra el curso de Estadística que vienen desarrollando los estudiantes de la Carrera Profesional de Enfermería de la Universidad Católica Los Ángeles de Chimbote.

\section{HIPÓTESIS}

\section{HIPÓTESIS GENERAL}

La aplicación del aprendizaje basado en problemas en la modalidad blended learning mejora significativamente el aprendizaje de las medidas de tendencia central y dispersión en los estudiantes de la carrera profesional de Enfermería de la Universidad Católica los Ángeles de Chimbote, semestre académico 2014- 02. 


\section{HIPÓTESIS ESPECÍFICAS}

La aplicación del aprendizaje basado en problemas en la modalidad blended learning mejora significativamente el aprendizaje de las medidas de tendencia central en los estudiantes de la carrera profesional de Enfermería de la Universidad Católica los Ángeles de Chimbote, semestre académico 2014 -02.

La aplicación del aprendizaje basado en problemas en la modalidad blended learning mejora significativamente el aprendizaje de las medidas de dispersión en los estudiantes de la carrera profesional de Enfermería de la Universidad Católica los Ángeles de Chimbote, semestre académico 2014 -02.

\section{METODOLOGÍA}

La presente investigación es de enfoque cuantitativa de corte transversal; de nivel explicativo.

Se utilizó el diseño de pretest - postest con un solo grupo.
G
$\mathrm{O}_{1}$
$\mathrm{X}$
$\mathrm{O}_{2}$

Donde:

G: grupo de estudiantes que participan en la investigación.

$\mathrm{O}_{1}$ : Logro del aprendizaje de medidas de tendencia central y dispersión obtenido en el pre-test

$\mathrm{O}_{2}$ : Logro del aprendizaje de medidas de tendencia central y dispersión en el post- test.

X: Aplicación de la Metodología del ABP en la modalidad blended learning.

Al grupo de estudiantes al inicio de la unidad se le aplica un pretest previa al estímulo o tratamiento, después se le administra el tratamiento (Propuesta didáctica) y finalmente se le aplica un postest posterior al estímulo.

La población está conformada por 26 estudiantes de la carrera profesional de Enfermería matriculados en la asignatura de Estadística - Sección A, en el semestre académico 2014-02 de la Universidad Católica los Ángeles de Chimbote.

Los criterios de inclusión fueron: Estudiantes matriculados por primera vez en la asignatura de estadística y estudiantes que asisten regularmente a las sesiones de clase. 
Se excluyen del estudio a los alumnos que llevan la asignatura por 2 o más veces.

Los datos se obtuvieron mediante la utilización de un conjunto de técnicas e instrumentos de evaluación que permitirán conocer el efecto de la aplicación de la variable independiente sobre la variable dependiente. Por ello, en la práctica de campo se ha empleado la técnica de la evaluación, y como instrumentos:

- Pre test y Post test, cuyo objetivo es evaluar el cumplimiento de los objetivos establecidos en el silabo y plan de aprendizaje.

- Rubricas de evaluación.

- Escalas de calificación para la evaluación del aprendizaje de las medidas de tendencia central y medidas de dispersión en blended learning.

Las puntuaciones de la escala de calificación de medidas de tendencia central y medidas de dispersión fueron obtenidas a través de la Baremación T.

BAREMOS T DE LA ESCALA DE CALIFICACIÓN DE MEDIDAS DE TENDENCIA CENTRAL Y MEDIDAS DE DISPERSIÓN

\begin{tabular}{ccc}
\hline $\begin{array}{c}\text { Escala y puntaje } \\
\text { estándar }\end{array}$ & Puntaje directo & $\begin{array}{c}\text { Escala de calificación de } \\
\text { medidas de tendencia central } \\
\text { y dispersión }\end{array}$ \\
\hline $70-+$ & $17-20$ & Excelente \\
$56-69$ & $14-16$ & Bueno \\
$41-55$ & $08-13$ & Regular \\
$31-40$ & $01-07$ & Malo \\
$00-30$ & 00 & Muy malo \\
\hline
\end{tabular}

Fuente: Elaboración propia.

Cabe resaltar que los instrumentos han sido validados por expertos, quienes han dado fe de la confiabilidad y validez de los mismos. Se calculó el coeficiente de Validez de Contenido por juicio de expertos (CVC) siendo este mayor a 0,80 lo cual conforma validez de la Escala de calificación para evaluar el aprendizaje de las medidas de tendencia central y dispersión.

El procesamiento de datos se realizó con el programa informático PASW Statistics 18, para el análisis de la información de utilizó la estadística descriptiva 
y para la contrastación de la hipótesis la prueba estadística no paramétrica de Wilcoxon con un nivel de significancia del $5 \%$.

\section{RESULTADOS}

Estimación del logro de aprendizaje respecto a las medidas de tendencia central

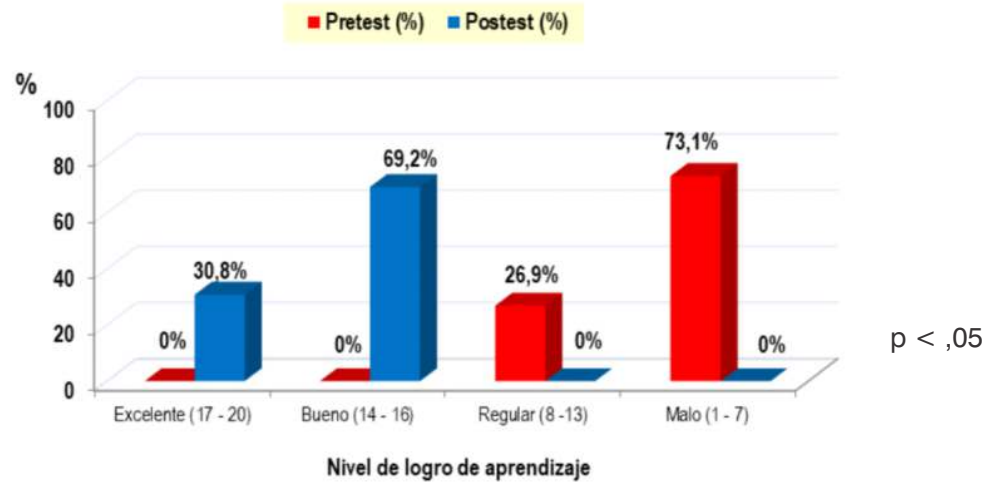

Figura 1. Nivel del logro de aprendizaje obtenido en el pretest y postest de medidas de tendencia central aplicado a los estudiantes de la carrera profesional de Enfermería de ULADECH Católica, en el semestre académico 2014-02.

Fuente: Pretest y postest aplicado a los estudiantes de Enfermería del II ciclo - "A".

Estimación del logro de aprendizaje respecto a las medidas de dispersión

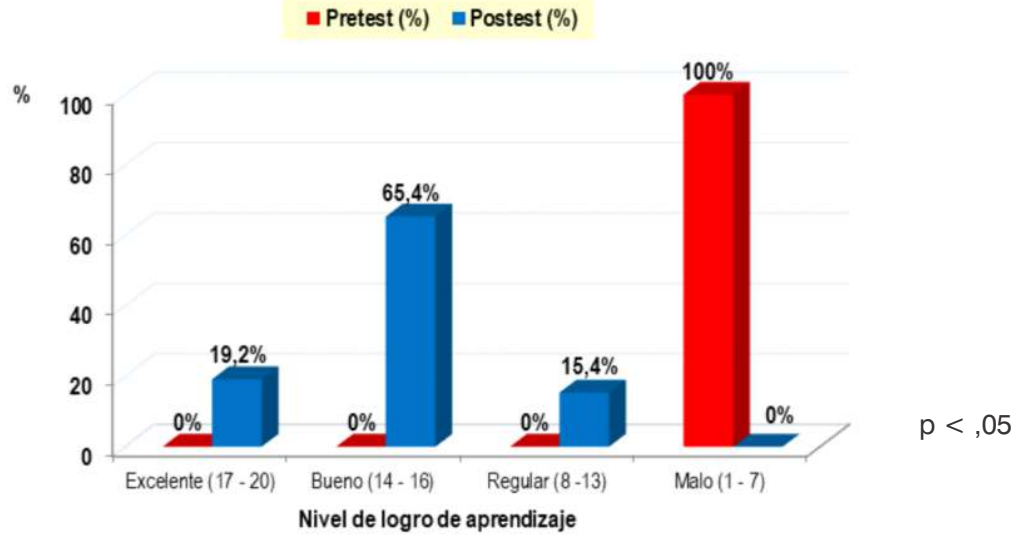

Figura 2. Nivel del logro de aprendizaje obtenido en el pretest y postest de medidas de dispersión aplicado a los estudiantes de la carrera profesional de Enfermería de ULADECH Católica, en el semestre académico 2014-02.

Fuente: Pretest y postest aplicado a los estudiantes de Enfermería del II ciclo - "A". 
Estimación del logro de aprendizaje respecto a las medidas de tendencia central y de dispersión

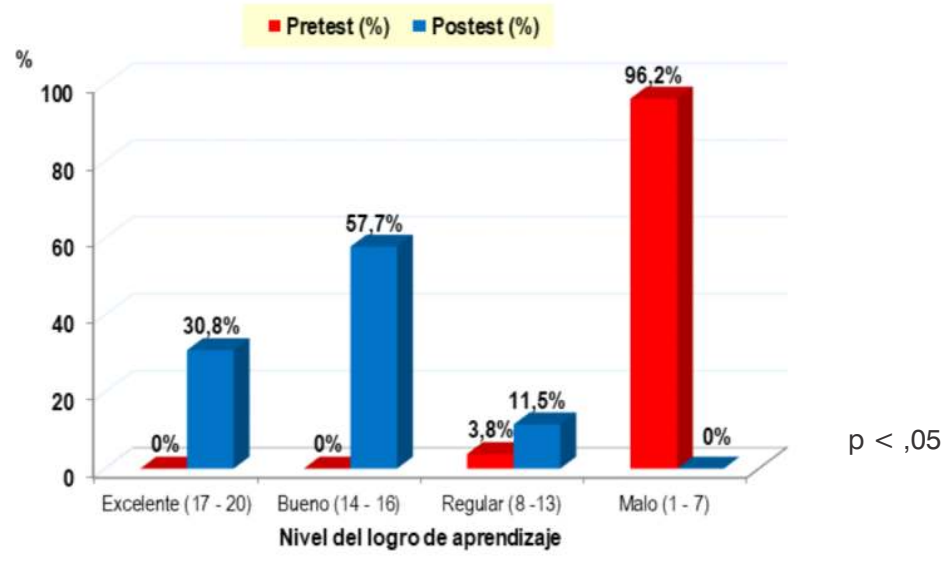

Figura 3. Nivel del logro de aprendizaje obtenidas en el pretest y postest de medidas de tendencia central y dispersión aplicado a los estudiantes de la carrera profesional de Enfermería de ULADECH Católica, en el semestre académico 2014-02.

Fuente: Pretest y postest aplicado a los estudiantes de Enfermería del II ciclo - "A".

\section{DISCUSIÓN}

Se procede a realizar el análisis de los resultados presentados anteriormente, con la finalidad de analizar el efecto de la aplicación de la variable independiente: Aplicación de estrategias didácticas en ABP con el uso del blended learning, sobre la variable dependiente: Aprendizaje de las medidas de tendencia central y medidas de dispersión.

\section{Evaluación del aprendizaje de las medidas de tendencia central en el pretest y postest}

En el diagnóstico realizado a los estudiantes respecto a las medidas de tendencia central se utilizó un pretest para determinar el nivel en el que se encontraban cada uno de los estudiantes.

Tal como se afirma en los resultados del pretest en la Figura 1, los estudiantes del segundo ciclo - "A" de la carrera profesional de enfermería demostraron tener un nivel malo en un $73,1 \%$ y regular un $26,9 \%$. Con estos resultados se demuestra 
que la mayoría de estudiantes tienen un desconocimiento respecto al cálculo, interpretación y análisis de las medidas de tendencia central (media aritmética, mediana y moda) a partir de datos obtenidos de su entorno profesional. Frente a estos resultados se procedió a ejecutar la propuesta de intervención educativa basada en la aplicación de estrategias didácticas en ABP con el uso del blended learning.

Posterior a aplicación de la propuesta de intervención educativa se obtuvo como resultado que el $30,8 \%$ de los estudiantes obtuvieron un nivel de excelente y el $69,2 \%$ un nivel bueno respecto al aprendizaje las medidas de tendencia central conforme se evidencia en Figura 1.

\section{Evaluación del aprendizaje de las medidas de dispersión en el pretest y postest}

En el diagnóstico realizado respecto a las medidas de dispersión (varianza, desviación estándar y coeficiente de variación) se utilizó un pretest para determinar el nivel en el que se encontraban cada uno de los estudiantes.

Tal como se muestra en los resultados del pretest en la Figura 2, los estudiantes del segundo ciclo - "A" de la carrera profesional de enfermería demostraron tener un nivel malo en un $100 \%$. Con estos resultados se demuestra que la totalidad de estudiantes que participaron en la presente investigación tienen un desconocimiento respecto al cálculo, interpretación y análisis de las medidas de dispersión a partir de datos obtenidos de su entorno profesional. Frente a estos resultados se procedió a ejecutar la propuesta de intervención educativa basada en la aplicación de estrategias didácticas en ABP con el uso del blended learning.

Posterior a aplicación de la propuesta de intervención educativa se obtuvo como resultado que el 19,2\% de los estudiantes obtuvieron un nivel de excelente y el $65,4 \%$ un nivel bueno respecto al aprendizaje las medidas de tendencia central y dispersión conforme se evidencia en Figura 2.

\section{Evaluación del aprendizaje de las medidas de tendencia central y dispersión en el pretest y postest}

En el diagnóstico realizado a los estudiantes respecto a las medidas de tendencia central y dispersión se utilizó un pretest para determinar el nivel en el que se encontraban cada uno de los estudiantes. 
Tal como se afirma en los resultados del pretest en la Figura 3, los estudiantes del segundo ciclo - "A" de la carrera profesional de enfermería demostraron tener un nivel malo en un $96,2 \%$ y regular un $3,8 \%$. Con estos resultados se demuestra que la mayoría de estudiantes tienen un desconocimiento respecto al cálculo, interpretación y análisis de las medidas de tendencia central y dispersión a partir de datos obtenidos de su entorno profesional. Frente a estos resultados se procedió a ejecutar la propuesta de intervención educativa basada en la aplicación de estrategias didácticas en ABP con el uso del blended learning.

Posterior a aplicación de la propuesta de intervención educativa se obtuvo como resultado que el $30,8 \%$ de los estudiantes obtuvieron un nivel excelente y el $57,7 \%$ un nivel bueno respecto al aprendizaje de las medidas de tendencia central conforme se evidencia en la Figura 3.

Estos resultados son corroborados por la investigación realizada por Villanueva y Moreno (2010) en su investigación "Aprendizaje basado en problemas y el uso de las Tic para el mejoramiento de la competencia interpretativa en estadística descripción: el caso de las medidas de tendencia central, donde se concluye que los procesos estadísticos que se venían desarrollando en la institución se manejaban de manera tradicional, a partir de la verbalización, repetición, memorización y aplicación algorítmica en forma mecánica. Para contribuir a desarrollar la competencia interpretativa, la enseñanza de la estadística debe aportar a los estudiantes procesos cognitivos y meta cognitivos que les permitan resolver problemas. En este sentido, el enfoque metodológico didáctico $\mathrm{ABP}$ y las mediciones tecnológicas utilizadas en la tesis, son un camino en esa dirección que ha demostrado su pertinencia y eficacia.

\section{Comparación de los resultados obtenidos en el pretest y postest}

Para comprobar las hipótesis de la investigación general y específica se utilizó la prueba estadística de Wilcoxon con un nivel de significancia del 5\%.

Para el caso de la comparación de las calificaciones obtenidas en el pretest y postest respecto a las medidas de tendencia central se obtuvo una significancia de 0,000 siendo este valor menor que 0,05. Este resultado indica que sí existe una diferencia significativa entre el logro de aprendizaje obtenido en el pretest con el logro del postest, evidenciándose que mejora el aprendizaje en el postest (Figura 1).

Respecto a la comparación de las calificaciones obtenidas en el pretest y postest de las medidas de dispersión se obtuvo una significancia de 0,000 siendo este valor menor que 0,05. Este resultado indica que sí existe una diferencia signi- 
ficativa entre el logro de aprendizaje obtenido en el pre test con el logro del postest, evidenciándose que mejora el aprendizaje en el postest (Figura 2).

Así mismo, en el contraste de la hipótesis general con la prueba de Wilcoxon se obtuvo un nivel de significancia de 0,000 valor menor a 0,05 ; lo cual evidencia que si hay una diferencia significativa entre las calificaciones obtenidas en el pretest y postest; siendo mayores las calificaciones obtenidas en el postest (Figura 3). Estos resultados confirman que la aplicación de la metodología del ABP en la modalidad blended learning contribuyó a mejorar el aprendizaje respecto a las medidas de tendencia central y dispersión en los estudiantes del segundo ciclo de la carrera profesional de Enfermería - Chimbote.

Espinoza y Sánchez (s.f.) en su investigación titulada "Aprendizaje basado en problemas para enseñar y aprender estadística y probabilidad" concluyen que los resultados obtenidos en la investigación muestran cambios estadísticamente significativos en algunas de las categorías analizadas como indicadores de aprendizaje significativo y una valoración favorable.

Los resultados encontrados refuerzan lo indicado por Rayser y otros (1995) quién afirma que hay un mayor grado de aprendizaje en el caso que se aplica la técnica basada en problemas en comparación con la docencia tradicional; más aún cuando la aplicación de esta técnica se apoya en nuevas tecnologías (Coley y otros, 1996).

\section{CONCLUSIONES}

Los resultados obtenidos en el pretest reflejaron que el 73,1\% de los estudiantes del segundo ciclo - "A" de la carrera profesional de Enfermería obtuvieron un nivel de logró de aprendizaje "Malo" respecto a las medidas de tendencia central y dispersión.

Los resultados obtenidos en el postest reflejaron que el 69,2\% de los estudiantes del segundo ciclo - "A" de la carrera profesional de Enfermería obtuvieron un nivel de logro de aprendizaje "Bueno" respecto a las medidas de tendencia central y dispersión.

La aplicación de estrategias didácticas en ABP con el uso del blended learning se realizó en 6 sesiones de aprendizaje.

Después de contrastar la hipótesis de investigación se determinó que si existe una diferencia significativa en el logro del aprendizaje obtenido entre el pretest 
y postest dando como resultado el valor de $\mathrm{p}=0,000<0,05$, esto demuestra que la aplicación de estrategias didácticas en $\mathrm{ABP}$ con el uso del blended learning ha contribuido en mejorar significativamente el aprendizaje de las medidas de tendencia central y dispersión.

\section{REFERENCIAS BIBLIOGRÁFICAS}

Coley, R.J., Cradler, J., and Engel, P.K. (1996). Computers and classrooms: The status of technology in U.S. schools (Policy information report). Princeton, NJ: Educational Testing Service.

Duch, B. Groh, S. and Allen, D. (2004). The power of problem-based learning: a practical 'how to' for teaching courses in any discipline. Sterling, VA: Stylus.

Espinoza, C. y Sanchez, I. Aprendizaje basado en problemas para enseñar y aprender estadística y probabilidad. Paradígma [online]. 2014, vol.35, n.1 [citado 2015-09-01] , pp. 103-128. Recuperado en: <http://www.scielo.org.ve/scielo.php?script=sci_arttext\&pid=S1011 $22512014000100005 \& \operatorname{lng}=$ es\&nrm $=$ iso $>$. ISSN 1011-2251.

Hinojo, Aznar, Cáceres (2009). Percepciones del alumnado sobre el blended learning en la universidad. Recuperado en: http://es.calameo.com/books/003296962c3b63e5efa41

Ryser, G.R., Beeler, J.E., and McKenzie, C.M. (1995). Effects of a Computer-Supported Intentional Learning Environment (CSILE) on students'self- concept, self-regulatory behavior, and critical thinking ability. Journal of Educational Computing Research 13(4), pp. 375-385.

Sánchez, I; Moreira, M; y Caballero, C. (2009). Implementación de una propuesta de aprendizaje significativo de la cinemática a través de la resolución de problemas. Ingeniare. Rev. Chil. Ing. 17 (1). 27-41.

Villanueva H. y Moreno M. (2010). Aprendizaje basado en problemas y el uso de las TIC para el mejoramiento de la competencia interpretativa en estadística descriptiva: El caso de las medidas de tendencia central (Tesis de maestría). Recuperado en: http://www.elitv.org/ documentos/tesis/tesis5TICparamejoramiento.pdf 